Kansas State University Libraries

New Prairie Press

\title{
MIXED MODELS APPROACH TO ON-FARM TRIALS: AN ALTERNATIVE TO META-ANALYSIS FOR COMPARING ONE TREATMENT TO POSSIBLY DIFFERENT CONTROLS
}

Peter M. Njuho

George A. Milliken

Follow this and additional works at: https://newprairiepress.org/agstatconference

Part of the Agriculture Commons, and the Applied Statistics Commons

\section{(c) (1) $\Theta(9$}

This work is licensed under a Creative Commons Attribution-Noncommercial-No Derivative Works 4.0 License.

\section{Recommended Citation}

Njuho, Peter M. and Milliken, George A. (1995). "MIXED MODELS APPROACH TO ON-FARM TRIALS: AN ALTERNATIVE TO META-ANALYSIS FOR COMPARING ONE TREATMENT TO POSSIBLY DIFFERENT CONTROLS," Conference on Applied Statistics in Agriculture. https://doi.org/10.4148/2475-7772.1343

This is brought to you for free and open access by the Conferences at New Prairie Press. It has been accepted for inclusion in Conference on Applied Statistics in Agriculture by an authorized administrator of New Prairie Press. For more information, please contact cads@k-state.edu. 


\title{
MIXED MODELS APPROACH TO ON-FARM TRIALS: AN ALTERNATIVE TO META-ANALYSIS FOR COMPARING ONE TREATMENT TO POSSIBLY DIFFERENT CONTROLS.
}

\author{
Peter M. Njuho and George A. Milliken \\ Department of Statistics \\ Kansas State University
}

\begin{abstract}
The estimator of effect size, the sample mean difference divided by the sample standard error of the difference is studied in the context of mixed models and is related to the analysis of on-farm trials. A single treatment is compared against possibly different controls using a completely randomized design on each farm. A lower $(1-\infty) 100 \%$ confidence limit on mean difference of the treatment and the average control is obtained. The best linear unbiased predictors (BLUPS) of the mean difference of the treatment and the individual controls as well as the lower $(1-\alpha) 100 \%$ prediction limits are provided. The effect of omitting or not omitting the farm-bytreatment interaction variance component in the weighting process is assessed using two numerical examples.
\end{abstract}

\section{Introduction}

On-farm trials are experiments conducted in farmers' fields, usually with the cooperation and participation of the farmers (Amir and Knipscheer, 1989). Farmers are allowed to evaluate the treatment ("new" technology) on their farms while it is compared to the farmers' control ("old" technology). It is unlikely that all farmers involved in the trial will have a common control. Lack of a common control makes it difficult to evaluate the treatment effect efficiently across the farms. A common practice is to use the same experimental design for all farms involved in the trial. Such practice fails to effectively control the known farm variations. For instance, one farm may require a completely randomized design, while another farm may require a randomized complete block design. The choice of the design to be used on a given farm depends on the nature of the within farm variation to be controlled. Therefore, using the same design structure on each farm may not be appropriate.

Mixed models and meta-analysis methods are used to extract the information from the individual trials and combine the information across trials. These methods are used to compare the treatment to the mean of the controls and to the individual controls through the construction of confidence intervals and prediction intervals.

Good experimental designs and the form of analyses appropriate to on-farm trials remain 
to be defined (Stucker and Hicks, 1993). Some groups of researchers do not regard any research conducted on farms as scientific whereas, many on-farm researchers disregard basic statistical principles. This paper provides a statistical procedure that allows for the treatment to be compared to possibly different controls on each farm while allowing for a different experimental design on each farm. The use of meta-analysis and mixed models methods are constructed in this on-farm trials setting.

\section{Model}

Suppose f farms have been randomly selected to be involved in an on-farm trial where a treatment is compared against the possibly different control on each farm. Assume that a completely randomized design (CRD) is used on each farm with the treatment and control each replicated $r$ times. Thus the following development assumes the same design is used at each farm but this requirement is removed in later sections. The use of the CRD is more simplistic than those used in practice, but the basic principles are not design-specific. Hence, the CRD simply introduces the main ideas.

A model to describe the response from the $j^{\text {th }}$ replication of the $k^{\text {th }}$ treatment from the $i^{\text {th }}$ farm is

$$
\begin{gathered}
Y_{i j k}=\mu+f_{i}+\tau_{k}+f_{i k}+\epsilon_{i j k} \\
\mathrm{i}=1,2, \ldots, \mathrm{f}, \mathrm{j}=1,2, \ldots, \mathrm{r}, \mathrm{k}=\mathrm{T}, \mathrm{C}_{i}
\end{gathered}
$$

where, $\mathrm{T}=$ Treatment and $\mathrm{C}_{i}=$ Control on $i^{\text {th }}$ farm, $Y_{i j k}$ is the observed response in the $j^{\text {th }}$ replication within the $i^{\text {th }}$ farm receiving the $k^{\text {th }}$ treatment, $\mu$ is the overall mean, $f_{i}$ is the $i^{\text {th }}$ farm effect, $\tau_{k}$ is the $k^{\text {th }}$ treatment effect, $f_{i k}$ is the interaction between the $i$ th farm and the $k^{\text {th }}$ treatment, and $\epsilon_{i j k}$ is the random error or experimental unit error.

Model (1) has three random effects with assumed distributions $f_{i} \sim$ iid $N\left(0, \sigma_{f}^{2}\right)$, $f_{i T} \sim i i d N\left(0, \sigma_{T T}^{2}\right), t_{i C_{i}} \sim i i d N\left(0, \sigma^{2} C_{C}\right)$ and $\varepsilon_{i j k}$ are independent normal with mean zero and variance $\sigma_{i}^{2}$. Model (1) can be written in terms as a treatment $(T)$ model and a set of control $\left(C_{i}\right.$, $\mathrm{i}=1,2, \ldots, \mathrm{f})$ models. The treatment model is

$$
Y_{i j T}=\mu_{T}+f_{i}+f t_{i T}+\epsilon_{i j T}
$$

where $\mu_{T}=\mu+\tau_{T}$. Let $U_{i T}=\mu_{T}+f_{i}+f_{i T}$ be the predictable function of treatment $T$ on farm $i$, then (2) can be expressed as

$$
Y_{i j T}=U_{i T}+\varepsilon_{i j T}
$$

Most often the farms used in on-farm trials are randomly selected, thus farmer's controls 
$\left(C_{1}, C_{2}, \ldots, C_{f}\right)$ are random variables distributed with mean $\mu_{c}$ and variance $\sigma_{c}^{2}$. The control models are

$$
Y_{i j c_{1}}=\mu_{c}+f_{i}+f t_{i c_{1}}+\epsilon_{i j c_{i}}, \quad i=1,2, \ldots, \mathrm{f}
$$

where $\mu_{c}=\mu$, and $U_{i c_{1}}=\mu_{c}+f_{i}+t_{i C_{1}}$ is the predictable function of control $C_{i}$ on farm i. Hence, (4) can be expressed as

$$
Y_{i j c_{1}}=U_{i c_{1}}+\epsilon_{i j c_{1}}
$$

The expectations and variances of $U_{i T}$ and $U_{i C_{1}}, i=1,2, \ldots, f$ are $E\left[U_{i T}\right]=\mu_{T}$ with $\operatorname{Var}\left(U_{i T}\right)=\sigma_{f}^{2}+\sigma_{\pi}^{2}$, and $E\left[U_{i c}\right]=\mu_{c}$ with $\operatorname{Var}\left(U_{i c}\right)=\sigma_{f}^{2}+\sigma_{r C}^{2}$.

To estimate the variance components $\sigma_{r T}^{2}$ and $\sigma_{r c}^{2}$, one would need multiple treatments and controls per farm and in this case we have neither. Thus inorder to have identifiable parameters for model (1), we assume $\sigma_{\pi}^{2}=\sigma_{r c}^{2}=\sigma_{\pi}^{2}$. Under this assumption, the random variables $U_{i T}$ and $U_{i C_{i}}, \mathrm{i}=1,2, \ldots$, f are distributed with different means $\mu_{T}$ and $\mu_{C}$ and a common variance, $\sigma_{f}^{2}+\sigma_{\pi}^{2}$. The meta-analysis framework uses standardized differences between the treatment and control means from each farm (Hedges, 1981). We define two measures of standardized mean difference as follows:

a) $\delta_{i T}^{\prime}=\left(U_{i T}-U_{i C}\right) / \sigma_{i}$ with $\operatorname{Var}\left(\delta_{i T}^{\prime}\right)=2 \sigma_{n}^{2} / \sigma_{i}^{2}$

b) $\delta_{i T}^{\prime \prime}=\left(U_{I T}-U_{i C_{C}}\right) / \sqrt{\operatorname{Var}\left(U_{i T}-U_{i C}\right)}$ with $\operatorname{Var}\left(\delta_{I T}^{\prime \prime}\right)=1$

where, $\operatorname{Var}\left(U_{i T}-U_{i C_{i}}\right)=2 \sigma_{\pi}^{2}, \mathrm{i}=1,2, \ldots, \mathrm{f}$.

The random variables $\delta_{i T}^{\prime}$ and $\delta_{i T}^{\prime \prime}$ differ by the weighting factor or divisor, where the former ignores the farm-by-treatment interaction variance component in the weighting process. The two random variables have a functional relation which is expressed as

$$
\begin{aligned}
\delta_{i T}^{\prime \prime} & =\left(U_{i T}-U_{i C}\right) / \sqrt{\operatorname{Var}\left(U_{i T}-U_{i C}\right)}=\left(U_{i T}-U_{i C}\right) / \sqrt{2 \sigma_{n}^{2}} \\
& =\frac{\left(U_{i T}-U_{i C}\right) / \sigma_{i}}{\sqrt{2 \sigma_{n}^{2} / \sigma_{i}^{2}}}=\frac{\delta_{i T}^{\prime}}{\sqrt{2 \sigma_{n}^{2} / \sigma_{i}^{2}}}
\end{aligned}
$$

This implies that $\delta_{i T}^{\prime \prime}=A_{i} \delta_{i T}^{\prime}$, where $A_{i}=\sqrt{\sigma_{i}^{2} / 2 \sigma_{n}^{2}}, \mathrm{i}=1,2, \ldots, \mathrm{f}$ and $\sigma_{n}^{2}>0$.

Equation (6) is commonly used in meta-analysis (Hedges and Olkin, 1985; Li, Shi, and Roth, 1994). It is usually denoted by $\delta_{i}=\left(\mu_{i}^{E}-\mu_{i}^{C}\right) / \sigma_{i}$ for the $i^{\text {th }}$ study where $\mu_{i}^{E}$ and $\mu_{i}^{C}$ are 
treatment and control means, respectively, with a common variance $\sigma_{i}^{2}$. The parameter $\delta$, defined by $\delta=E\left(\delta_{i}\right), i=1,2, \ldots, f$ is known as the standardized mean difference or effect size (Hedges and Olkin 1985). Hedges and Olkin did not incorporate the study-by-treatment interaction variance component in their weighting process because, generally in meta-analysis the responses measured could differ from one study to another. Hence, the study-by-treatment interaction variance component would not be interpretable.

In our case, the farm-by-treatment interaction variance component is included. Therefore, the discussion that follows is based on (7). Towards the end of the presentation, (6) and (7) are compared using examples which help to determine the effect of omitting $\sigma_{f t}^{2}$ in the weighting process.

\subsection{Prediction of $\delta_{i T}^{\prime \prime}$}

Equations (3) and (5) are used for the prediction of both $U_{i T}$ and $U_{i C_{i}}$. The means $\bar{Y}_{. T}$ and $\bar{Y}_{. . C}$ are unbiased estimators of $\mu_{T}$ and $\mu_{C}$, and $\bar{Y}_{i, T}$ and $\bar{Y}_{i, C_{i}}$ are predictors of $U_{i T}$ and $U_{i C_{i}}, i=1,2, \ldots, f$ which are represented by $\tilde{U}_{i T}=\bar{Y}_{i, T}$ and $\tilde{U}_{i C_{i}}=\bar{Y}_{i, C_{i}}$. A model describing the differences between the treatment and control means from the $i$ th farm is constructed as

$$
\begin{aligned}
S_{i}= & \tilde{U}_{i T}-\tilde{U}_{i C_{i}} \\
= & \mu_{T}-\mu_{C}+f_{i T}-\tilde{t}_{i C_{i}}+\bar{\epsilon}_{i, T}-\bar{\epsilon}_{i, C_{i}} \\
& \mathrm{i}=1,2, \ldots, \mathrm{f}
\end{aligned}
$$

Equation (9) can be expressed in a compact form as

$$
S_{i}=\mu_{s}+t_{i}+e_{i}
$$

where, $\mu_{s}=\mu_{T}-\mu_{C}, t_{i}=f_{i T}-f_{i C_{i}}$ and $\epsilon_{i}=\bar{\varepsilon}_{i, T}-\bar{\varepsilon}_{i, C_{i}}, \mathrm{i}=1,2, \ldots, \mathrm{f}$. Model (10) is a mixed model with fixed effect $\mu_{s}$ and random effects $t_{i}$ and $\varepsilon_{i}, i=1,2, \ldots$, f. The expectation and variance of the random variables $S_{i}, i=1,2, \ldots, f$ are $E\left[S_{i}\right]=E\left[\tilde{U}_{i T}-\tilde{U}_{i C}\right]=\mu_{T}-\mu_{C}$ with $\operatorname{Var}\left[S_{i}\right]=2\left(\sigma_{\tilde{n}}^{2}+\sigma_{i}^{2} / n\right)$ and $\operatorname{Cov}\left(S_{i}, S_{i}\right)=0, \quad \forall_{j} ; \neq i^{\prime}$. Hence, the random variables $S_{1}, S_{2}, \ldots, S_{f}$ are independently distributed with mean $\mu_{s}$ and variance $2\left(\sigma_{f t}^{2}+\sigma_{j}^{2} / n\right.$.

The sample standardized mean difference $d_{i T}, \mathrm{i}=1,2, \ldots, \mathrm{f}$ is the predictor of $\delta_{i T}^{\prime \prime}$ and is expressed as

$$
\begin{aligned}
& d_{i T}=\left(\tilde{U}_{i T}-\tilde{U}_{i C}\right) / \sqrt{\operatorname{Var}\left(\tilde{U}_{i T}-\tilde{U}_{i C}\right)} \\
& \mathrm{i}=1,2, \ldots, \mathrm{f}
\end{aligned}
$$


To estimate the parameters, we express the model (10) in matrix notation as

$$
\underline{s}=i_{f} \mu_{s}+\mathbb{I}_{f} \underline{t}+\underline{\varepsilon}
$$

where, $\underline{S}=\left(S_{1}, S_{2}, \ldots, S_{f}\right)^{\prime}, \underline{t}=\left(t_{1}, t_{2}, \ldots, t_{f}\right)^{\prime}, \underline{\varepsilon}=\left(\varepsilon_{1}, \epsilon_{2}, \ldots, \varepsilon_{f}\right)^{\prime}, I_{f}$ is an fxf identity matrix and $i_{f}$ is an fxi vector of ones. By letting $i_{f}=X$ and $I_{f}=Z$, (12) can be expressed in terms of $\mathbf{X}$ and $\mathbb{Z}$ as $\underline{\underline{S}}=\underline{X} \mu_{s}+\underline{Z} \underline{t}+\underline{\varepsilon}$ where, $E[\underline{S}]=X \mu_{s}$ with $\operatorname{Var}[\underline{S}]=Z \operatorname{Var}[\underline{t}+\underline{\varepsilon}] Z^{\prime}$. Let, $\Delta=\operatorname{Var}[\underline{S}], G=\operatorname{Var}[\underline{t}]$ and $R=\operatorname{Var}[\underline{\varepsilon}]$, then $\Delta=Z G Z^{\prime}+R$. Thus, $\Delta$ is an fxf diagonal matrix of unknown variance components, that is, $\Delta=2 \operatorname{diag}\left\{\sigma_{f t}^{2}+\sigma_{1}^{2} / r, \sigma_{f t}^{2}+\sigma_{2}^{2} / r, \ldots, \sigma_{f t}^{2}+\sigma_{f}^{2} / r\right\}$.

If $\Delta$ is a matrix of known variance components, the best linear unbiased estimator (BLUE) of $\mu_{s}$ can be computed as

$$
\begin{aligned}
\hat{\mu}_{s} & =\left(X^{\prime} \Delta^{-1} X\right)^{-1} X^{\prime} \Delta^{-1} \underline{s} \\
& =\left(i_{f}^{\prime} \Delta^{-1} i_{f}\right)^{-1} i_{f}^{\prime} \Delta^{-1} \underline{S} \\
& =\left[\sum_{i=1}^{f}\left(2\left(\sigma_{\tilde{\pi}}^{2}+\sigma_{i}^{2} / r\right)\right)^{-1}\right]^{-1} \sum_{i=1}^{f} s_{i} / 2\left(\sigma_{\tilde{t}}^{2}+\sigma_{i}^{2} / r\right)
\end{aligned}
$$

The variance of $\hat{\mu}_{s}$ in $(13)$ is $\operatorname{Var}\left(\hat{\mu}_{s}\right)=\left(X^{\prime} \underline{\Delta}^{-1} X\right)^{-1}=\left[\sum_{i=1}^{f}\left(2\left(\sigma_{f t}^{2}+\sigma_{i}^{2} / n\right)^{-1}\right]^{-1}\right.$.

If $\Delta$ is a matrix of unknown variance components, an estimator of $\mu_{s}$ can also be obtained by substituting $\hat{\Delta}$ for $\Delta$ ( a matrix of estimates of variance components) in (13). The substitution does not alter the expected value of $\hat{\mu}_{s}$ but does have an effect on standard error (S.E.) of $\hat{\mu}_{s}$ (Kacker and Harville, 1984). Kacker and Harville (1984) provide a procedure for approximating the standard error of $\hat{\mu}_{s}$ and hence,

$$
\begin{aligned}
\hat{\mu}_{s} & =\left(X^{\prime} \hat{\Delta}^{-1} X\right)^{-1} X^{\prime} \hat{\Delta}^{-1} \underline{S} \\
& =\left(i_{f}^{\prime} \hat{\Delta}^{-1} i_{f}\right)^{-1} i_{f}^{\prime} \hat{\Delta}^{-1} \underline{S} \\
& =\left[\sum_{i=1}^{f}\left(2\left(\hat{\sigma}_{\tilde{t}}^{2}+\hat{\sigma}_{i}^{2} / r\right)\right)^{-1}\right]^{-1} \sum_{i=1}^{p} S_{i} / 2\left(\hat{\sigma}_{\tilde{t}}^{2}+\hat{\sigma}_{i}^{2} / r\right)
\end{aligned}
$$

The variance of $\hat{\mu}_{s}$ in $(14)$ is $\operatorname{Var}\left(\hat{\mu}_{s}\right)=\left(X^{\prime} \hat{\Delta}^{-1} X\right)^{-1}=\left[\sum_{i=1}^{f}\left(2\left(\hat{\sigma}_{\hat{f}}^{2}+\hat{\sigma}_{i}^{2} / r\right)\right)^{-1}\right]^{-1}$.

According to Kacker and Harville (1984), the estimated standard error $S . \hat{E} \cdot\left(\hat{\mu}_{s}\right)=\sqrt{\operatorname{Var}\left(\hat{\mu}_{s}\right)}$ underestimates the actual standard error. A more conservative estimate of the standard error can be computed using their approach. The estimator $\hat{\mu}_{s}$ given in (13) is related to the $d_{i T}$ in (11) as 


$$
\begin{aligned}
\hat{\mu}_{s} & =\left[\sum_{i=1}^{f}\left(2\left(\sigma_{i t}^{2}+\sigma_{i}^{2} / n\right)\right)^{-1}\right]^{-1} \sum_{i=1}^{f} s_{i} / 2\left(\sigma_{\tilde{t}}^{2}+\sigma_{i}^{2} / \eta\right) \\
& =\left[\sum_{i=1}^{f}\left(2\left(\sigma_{n t}^{2}+\sigma_{i}^{2} / n\right)\right)^{-1}\right]^{-1} \sum_{i=1}^{f} d_{i T} / \sqrt{2\left(\sigma_{i t}^{2}+\sigma_{i}^{2} / \eta\right)}
\end{aligned}
$$

Equation (15) shows the relationship between $\hat{\mu}_{s}$ and the $d_{i T}, i=1,2, \ldots, f$.

Let $\sigma_{p}^{2}=\left[\sum_{i=1}^{f}\left(2\left(\sigma_{f t}^{2}+\sigma_{i}^{2} / n\right)\right)^{-1}\right]^{-1}$ denote the variance of the estimate $\hat{\mu}_{s}$ when farm-bytreatment interaction variance component is included in the weighting process and $\sigma_{u}^{2}=\left[\sum_{i=1}^{f}\left(2 \sigma_{i}^{2} / \eta^{-1}\right]^{-1}\right.$ when it is not. Suppose $\hat{\sigma}_{p}^{2}$ and $\hat{\sigma}_{u}^{2}$ denote the estimates of $\sigma_{p}^{2}$ and $\sigma_{u}^{2}$, respectively. It holds true that $\frac{2 \hat{\sigma}_{\min }^{2}}{r f} \leq \hat{\sigma}_{u}^{2}$ and $\frac{2\left(\hat{\sigma}_{f t}^{2}+\hat{\sigma}_{\min }^{2}\right)}{\text { if }} \leq \hat{\sigma}_{p}^{2}$ where $\hat{\sigma}_{\min }^{2}=\min \left\{\hat{\sigma}_{1}^{2}, \hat{\sigma}_{2}^{2}, \ldots, \hat{\sigma}_{f}^{2}\right\}$.

\section{Lemma 1}

Assume $\hat{\sigma}_{\hat{t}}^{2}>0$ and $\hat{\sigma}_{i}^{2}>0, i=1,2, \ldots, f$, then $\hat{\sigma}_{u}^{2} \leq \hat{\sigma}_{p}^{2}$

\section{Proof of Lemma 1}

Clearly $2\left(\hat{\sigma}_{f t}^{2}+\hat{\sigma}_{i}^{2} / r\right) \geq 2 \hat{\sigma}_{i}^{2} / r$, where $\hat{\sigma}_{f t}^{2}>0, \hat{\sigma}_{i}^{2}>0$ and $i=1,2, \ldots, f$. It follows that $\left[2\left(\hat{\sigma}_{\tilde{t}}^{2}+\hat{\sigma}_{i}^{2} / r\right)\right]^{-1} \leq\left[2 \hat{\sigma}_{j}^{2} / r\right]^{-1}, \mathrm{i}=1,2, \ldots, \mathrm{f}$ which is equivalent to $\sum_{i=1}^{f}\left(2\left(\hat{\sigma}_{\tilde{r}}^{2}+\hat{\sigma}_{i}^{2} / r\right)\right)^{-1} \leq \sum_{i=1}^{f}\left(2 \hat{\sigma}_{i}^{2} / r\right)^{-1}$ This implies that $\left[\sum_{i=1}^{f}\left(2\left(\hat{\sigma}_{\tilde{t}}^{2}+\hat{\sigma}_{i}^{2} / r\right)\right)^{-1}\right]^{-1} \geq\left[\sum_{i=1}^{f}\left(2 \hat{\sigma}_{i}^{2} / r\right)^{-1}\right]^{-1}$ and hence, $\hat{\sigma}_{u}^{2} \leq \hat{\sigma}_{p}^{2}$.

The variances $\sigma_{p}^{2}$ and $\sigma_{u}^{2}$ are comprised of the weights used in the weighting process to compute $\hat{\mu}_{s}$ when $\sigma_{f t}^{2}$ is included and not included, respectively. The estimate $\hat{\sigma}_{u}^{2}$ which corresponds to the use of (6) is reported to be smaller than the minimum of the estimates of variances of individual studies or farms ( $\mathrm{Li}, \mathrm{Shi}$, and Roth, 1994). The estimate $\hat{\sigma}_{p}^{2}$ corresponds to the use of (7). Based on results of Lemma 1, the estimate $\hat{\sigma}_{p}^{2}$ is more conservative and less sensitive to the minimum of the estimates of the variances of individual farms than $\hat{\sigma}_{u}^{2}$.

In the estimation of $\mu_{s}$ and computation of its variance, several cases can be considered by either making assumptions concerning variances $\sigma_{i}^{2}, \mathrm{i}=1,2, \ldots, \mathrm{f}$ or the number of replications $r_{i}$ or both. A few of these cases are highlighted below. 


\subsection{Case I: Equal Numbers of Replications and Equal Variances}

Suppose the variances $\sigma_{i}^{2}, \mathrm{i}=1,2, \ldots$, f are equal to the unknown variance $\sigma^{2}$. Assume the farm-by-treatment interaction variance component, $\sigma_{f t}^{2}$, to be greater than zero. Consider an equal number of replications case and a possibly different design on each farm. The unknown variance components, $\sigma_{f t}^{2}$ and $\sigma^{2}$ are replaced by their estimators and hence (14) reduces to

$$
\begin{aligned}
\hat{\mu}_{s} & =\left[\sum_{i=1}^{f}\left(2\left(\hat{\sigma}_{\tilde{f} t}^{2}+\hat{\sigma}^{2} / r\right)\right)^{-1}\right]^{-1} \sum_{i=1}^{f} S_{i} / 2\left(\hat{\sigma}_{\tilde{t}}^{2}+\hat{\sigma}^{2} / r\right) \\
& =(1 / f) \sum_{i=1}^{f} S_{i}=\bar{Y}_{. . T}-\bar{Y}_{. C .}
\end{aligned}
$$

The variance of $\hat{\mu}_{s}$ in $(16)$ is $\operatorname{Var}\left(\hat{\mu}_{s}\right)=(2 / f)\left(\hat{\sigma}_{\tilde{t}}^{2}+\hat{\sigma}^{2} / n\right)$.

\subsection{Case II: Unequal Numbers of Replications and Equal Variances}

We have the same conditions as those in case I except that the number of replications at each farm are not equal thus, (14) can be written as

$$
\begin{aligned}
\hat{\mu}_{s} & =\left[\sum_{i=1}^{f}\left(2\left(\hat{\sigma}_{f t}^{2}+\hat{\sigma}^{2} / r_{i}\right)\right)^{-1}\right]^{-1} \sum_{i=1}^{f} S_{i} / 2\left(\hat{\sigma}_{\tilde{f}}^{2}+\hat{\sigma}^{2} / r_{i}\right) \\
& =\left[\sum_{i=1}^{f}\left(2\left(\hat{\sigma}_{f t}^{2}+\hat{\sigma}^{2} / r_{i}\right)\right)^{-1}\right]^{-1} \sum_{i=1}^{f}\left(\bar{Y}_{i, T}-\bar{Y}_{i, C_{i}}\right) / 2\left(\hat{\sigma}_{f t}^{2}+\hat{\sigma}^{2} / r_{i}\right)
\end{aligned}
$$

The variance of $\hat{\mu}_{s}$ in $(17)$ is $\operatorname{Var}\left(\hat{\mu}_{s}\right)=\left[\sum_{i=1}^{f}\left(2\left(\hat{\sigma}_{f t}^{2}+\hat{\sigma}^{2} / r_{i}\right)\right)^{-1}\right]^{-1}$.

The estimates $\hat{\sigma}_{f t}^{2}$ and $\hat{\sigma}^{2}$ are obtained either through maximum likelihood (ML), restricted maximum likelihood (REML), or method of moments (MM). These estimates are then used in the computation of $\hat{\mu}_{s}$ and its estimated variance.

\subsection{Case III: Unequal Numbers of Replications and Unequal Variances}

Suppose $\sigma_{i}^{2}, i=1,2, \ldots, \mathrm{f}$ are different and unknown. Assume unequal number of replications case and a possibly different design on each farm. A single treatment is compared against possibly different controls. As stated in case $\mathbb{I}$, the variance components $\sigma_{f t}^{2}$ and $\sigma_{i}^{2}$, $\mathrm{i}=1,2, \ldots, \mathrm{f}$ are replaced by their estimators. These estimates are substituted in (14) and thus

$$
\hat{\mu}_{s}=\left[\sum_{i=1}^{f}\left(2\left(\hat{\sigma}_{\hat{t}}^{2}+\hat{\sigma}_{i}^{2} / r_{i}\right)\right)^{-1}\right]^{-1} \sum_{i=1}^{f} S_{i} / 2\left(\hat{\sigma}_{\tilde{f} t}^{2}+\hat{\sigma}_{i}^{2} / r_{i}\right)
$$




$$
=\left[\sum_{i=1}^{f}\left(2\left(\hat{\sigma}_{i t}^{2}+\hat{\sigma}_{i}^{2} / r_{i}\right)\right)^{-1}\right]^{-1} \sum_{i=1}^{f}\left(\bar{Y}_{i, T}-\bar{Y}_{i, C_{j}}\right) / 2\left(\hat{\sigma}_{\tilde{i t}}^{2}+\hat{\sigma}_{i}^{2} / r_{i}\right)
$$

The variance of $\hat{\mu}_{s}$ in $(18)$ is $\operatorname{Var}\left(\hat{\mu}_{s}\right)=\left[\sum_{i=1}^{f}\left(2\left(\hat{\sigma}_{f t}^{2}+\hat{\sigma}_{j}^{2} / r_{j}\right)\right)^{-1}\right]^{-1}$.

The within farm variance components are estimated by $\hat{\sigma}_{1}^{2}=M S E_{1}, \hat{\sigma}_{2}^{2}=M S E_{2}, \ldots$, $\hat{\sigma}_{f}^{2}=M S E_{f}$ which are the within farm error mean squares and the farm by treatment variance component is estimated by $\hat{o}_{f t}^{2}=($ MSF $*$ TRT - MSE) $/ r$.

\subsection{A Lower $(1-\alpha) 100 \%$ Confidence Limit on $\mu_{T}-\mu_{C}$}

To determine if the treatment performs better than the population of farmers' controls, a lower $(1-\alpha) 100 \%$ confidence limit on the mean difference between the treatment and the average control, $\mu_{T}-\mu_{C}$ is obtained. An equal cost for observing a treatment and the control is assumed. Suppose $\hat{\mu}_{T}-\hat{\mu}_{C}, i=1,2, \ldots$, fare random variables distributed normal with mean $\mu_{T}-\mu_{C}$, and variance $\operatorname{Var}\left(\hat{\mu}_{T}-\hat{\mu}_{C}\right)$ as in case III. A lower $(1-\alpha) 100 \%$ confidence limit on the mean difference of the treatment and the average control is $\left(\hat{\mu}_{T}-\hat{\mu}_{C}\right)-t_{\alpha, v} \sqrt{\operatorname{Var}\left(\hat{\mu}_{T}-\hat{\mu}_{C}\right)}$ where $t_{\alpha, v}$ is the $\mathrm{t}$-value obtained at $\alpha$-level with $v$ degrees of freedom obtained using Satterthwaite's (1941) approximation procedure as

$$
V \approx \frac{2\left(\left[\sum_{i=1}^{f}\left(2\left(\hat{\sigma}_{f t}^{2}+\hat{\sigma}_{i}^{2} / r_{i}\right)\right)^{-1}\right]^{-1}\right)^{2}}{\left[\operatorname{approxi} \operatorname{Var}\left(\left[\sum_{i=1}^{f}\left(2\left(\hat{\sigma}_{f t}^{2}+\hat{\sigma}_{i}^{2} / r_{i}\right)\right)^{-1}\right]^{-1}\right]\right.} .
$$

The approximated variance of $\left[\sum_{i=1}^{f}\left(2\left(\hat{\sigma}_{f t}^{2}+\hat{\sigma}_{i}^{2} / r_{i}\right)\right)^{-1}\right]^{-1}$ can be obtained using the method discussed by Giesbrecht and Burn (1985). A simpler approach is to bound the variance and then use the approximated degrees of freedom corresponding to the bounds. One can easily show that $(2 / f)\left(\hat{\sigma}_{f t}^{2}+\hat{\sigma}_{\text {min }}^{2}\right) \leq\left[\sum_{i=1}^{f}\left(2\left(\hat{\sigma}_{f t}^{2}+\hat{\sigma}_{i}^{2} / r_{i}\right)^{-1}\right]^{-1} \leq(2 / f)\left(\hat{\sigma}_{f t}^{2}+\hat{\sigma}_{\text {max }}^{2}\right)\right.$ where, $\hat{\sigma}_{\text {min }^{*}}^{2}=\min \left\{\hat{\sigma}_{1}^{2} / r_{1}, \hat{\sigma}_{2}^{2} / r_{2}, \ldots, \hat{\sigma}_{f}^{2} / r_{f}\right\}$ and $\hat{\sigma}_{\text {max }}^{2}=\max \left\{\hat{\sigma}_{1}^{2} / r_{1}, \hat{\sigma}_{2}^{2} / r_{2}, \ldots, \hat{\sigma}_{f}^{2} / r_{f}\right\}$. The degrees of freedom corresponding to $(2 / f)\left(\hat{\sigma}_{t t}^{2}+\hat{\sigma}_{\text {min }^{*}}^{2}\right)$ and $(2 / f)\left(\hat{\sigma}_{f t}^{2}+\hat{\sigma}_{\text {max }^{*}}^{2}\right)$ are denoted by $v_{\text {min }^{*}}$ and $v_{\text {max }}$, respectively, and are approximated using the Satterthwaite's approximation as follows 


$$
\begin{aligned}
v_{\min ^{*}}= & \frac{\left[\hat{\eta}_{\min }\right]^{2}}{\frac{\left[a_{1} M S F * T R\right]^{2}}{f-1}+\left(\frac { a _ { 2 } ^ { 2 } } { 2 ( r - 1 ) } \left(\left[M S E_{1}\right]^{2}+\ldots+\left[M S E_{\min -1}\right]^{2}+\left[M S E_{\min +1}\right]^{2}+\right.\right.} \\
& \left.\left.\ldots+\left[M S E_{f}\right]^{2}\right)+\frac{1}{2(r-1)}\left[a_{3} M^{2} E_{\min }\right]^{2}\right)
\end{aligned}
$$

and

$$
\begin{aligned}
v_{\text {max }^{*}}= & \frac{\left[\hat{\eta}_{\max }\right]^{2}}{\frac{\left[a_{1} M S F * T R\right]^{2}}{f-1}+\left(\frac { a _ { 2 } ^ { 2 } } { 2 ( r - 1 ) } \left(\left[M S E_{1}\right]^{2}+\ldots+\left[M S E_{\max -1}\right]^{2}+\left[M S E_{\max +1}\right]^{2}+\right.\right.} \\
& \left.\left.\ldots+\left[M S E_{p}\right]^{2}\right)+\frac{1}{2(r-1)}\left[a_{3} M S E_{\max }\right]^{2}\right)
\end{aligned}
$$

where

$$
\begin{aligned}
& \hat{\eta}_{\min }=a_{1} M S F * T R T+a_{2}\left(M S E_{1}+M S E_{2}+\ldots+M S E_{\min -1}+M S E_{\min +1}+\ldots+M S E_{f}\right)+a_{3} M S E_{\min }, \\
& \hat{n}_{\max }=a_{1} M S F * T R T+a_{2}\left(M S E_{1}+M S E_{2}+\ldots+M S E_{\max -1}+M S E_{\max +1}+\ldots+M S E_{f}\right)+a_{3} M S E_{\max }, \\
& \hat{\sigma}_{p t}^{2}=\frac{(M S F * T R T-M S E)}{r}, a_{1}=\frac{2}{r}, a_{2}=-\frac{2}{r f}, \text { and } a_{3}=\frac{2(f-1)}{r f} .
\end{aligned}
$$

The degrees of freedom for the variance of $\hat{\mu}_{T}-\hat{\mu}_{C}$ can be approximated by the average of $v_{\min ^{*}}$ and $v_{\max ^{*}}$ as $v_{a v e r}=\left(v_{\min ^{*}}+v_{\max ^{*}}\right) / 2$. The average approximated degrees of freedom, $v_{a v e r}$ is almost equal to the farm-by-treatment interaction degrees of freedom for an equal variance and equal number of replications case. Therefore, it would be appropriate to use the farm-by-treatment interaction degrees of freedom to compute the confidence limit.

\subsection{A Lower (1- $\alpha) 100 \%$ Prediction Limit on $U_{i T}-U_{i C_{i}}$}

Most often, a farmer will be interested in knowing the predicted performance of the treatment, denoted by the predictable function $U_{i T}$ as compared to the predicted performance of the control on the farm, denoted by the predictable function $U_{i C_{i}}$. The BLUPS, $\tilde{U}_{i T}-\tilde{U}_{i C_{i}}, \mathrm{i}=1,2$, ..., f and their lower $(1-\alpha) 100 \%$ prediction limits are computed to enable each farmer to decide on adoption of the treatment. We want to predict the response of a farmer's control and compare it to the predicted response of the treatment on the farm. The predictable function for farm is $p_{i}=U_{i T}-U_{i C_{i}}=\mu_{T}-\mu_{C}+f_{i T}-f_{i C_{i}}, \mathrm{i}=1,2, \ldots, \mathfrak{f}$. The BLUP of $\rho_{i}$ is $\tilde{\rho}_{i}=\tilde{U}_{i T}-\tilde{U}_{i C_{i}}=\bar{Y}_{i . T}-\bar{Y}_{i C_{i}}=S_{i}$. A lower $(1-\alpha) 100 \%$ prediction limit on $U_{i T}-U_{i C_{i}}$ that allows for decision to be made for each farmer, assuming equal number of replications is $\left(\bar{Y}_{i, T}-\bar{Y}_{i, C_{i}}\right)-t_{\alpha, v_{i}} \sqrt{2\left(\hat{\sigma}_{i t}^{2}+\hat{\sigma}_{i}^{2} / r\right)}, \mathrm{i}=1,2, \ldots, \mathrm{f}$. Let denote a linear combination of the mean squares by $\hat{\eta}_{j}$. Thus, 


$$
\begin{aligned}
\hat{\eta}_{i} & =2\left(\hat{\sigma}_{\tilde{f}}^{2}+\hat{\sigma}_{i}^{2} / r\right) \\
& =a_{1} M S F * T R T+a_{2}\left(M S E_{i}+M S E_{2}+\ldots+M S E_{i-1}+M S E_{i+1}+\ldots+M S E_{f}\right)+a_{3} M S E_{i}
\end{aligned}
$$

Using the Satterthwaite's approximation, $v_{i} \hat{n}_{i} /\left(2\left(\sigma_{f i}^{2}+\sigma_{i}^{2} / n\right)\right), i=1,2, \ldots ., f$ is approximately distributed as a chi-square random variable with $v_{j}$ degrees of freedom where

$$
\begin{gathered}
v_{i}=\frac{\left[\hat{\eta}_{i}\right]^{2}}{\left[a_{1} M S F * T R\right]^{2}} \frac{a_{2}^{2}}{f-1}\left(\left[M S E_{1}\right]^{2}+\ldots+\left[M S E_{i-1}\right]^{2}+\left[M S E_{i+1}\right]^{2}+\right. \\
\left.\left.\ldots+\left[M S E_{i}\right]^{2}\right)+\frac{1}{2(r-1)}\left[a_{3} M S E_{i}\right]^{2}\right)
\end{gathered}
$$

\section{Examples}

\subsection{Example 1}

Consider a case where we have six farms, five replications per farm in a completely randomized design (CRD) and a single treatment along with the farmer's control. The treatment is compared against possibly different controls where the response is the yield of sorghum in bushels per acre. The 1991 Sorghum data given in Table 1 were obtained from Cooperative Extension Service Program, Department of Agronomy, Kansas State University.

The method of moments estimates of variance components are given in Table 2. The farms, replications and treatments are denoted by F, Rep and Trt, respectively. These variance component estimates were used in the computation of the estimate of $\mu_{s}$.

Table 3 shows summarized results of the estimation of $\mu_{s}$ computed in four ways. Combining and computing with equal and unequal variances, and when the estimate of farm-bytreatment interaction variance component, $\hat{o}_{f t}^{2}$, was either omitted or not omitted. The estimates given in Table 3 were computed using (16) and (18).

For the equal variance case, the estimate $\hat{\mu}_{s}$ is the same when $\hat{\sigma}_{\hat{n}}^{2}$ is either omitted or not omitted, but the estimated variances are different. These results indicate that for both equal and unequal variance cases, the estimated variance of $\hat{\mu}_{s}$ is smaller when the estimate of farm-bytreatment interaction variance component is omitted in the weighting process than when it is not. These results are in agreement with Lemma 1. From Table 3, for unequal variance and $\hat{\sigma}_{f t}^{2}$ not omitted $\hat{\mu}_{s}=5.02, S . \hat{E} \cdot\left(\hat{\mu}_{s}\right)=10.016$, the approximated degrees of freedom are

$$
v_{\text {aver }}=\left(v_{\text {min }^{*}}+v_{\text {max }^{*}}\right) / 2=(4.32+5.805) / 2=5.062 \approx 5 \text {, and } t_{.05,5.062}=2.010 \text {. Thus, a lower } 95 \%
$$
confidence limit on $\mu_{T}-\mu_{C}$ is $5.02-(2.010)(10.016)=-15.11$. The treatment fails to perform better than the mean of the population of farmers' controls since the lower $95 \%$ confidence limit on $\mu_{T}-\mu_{C}$ is not greater than zero. 
Table 4 provides the computed linear combination of the mean squares $\hat{\eta}_{\text {, }}$, the corresponding approximated degrees of freedom $v_{i}$, the approximated $t$-values at $\alpha=0.05$, the BLUPS of $U_{\pi T}-U_{i C_{i}}, \mathrm{i}=1,2, \ldots, \mathrm{f}$ and the estimated standard errors, and the lower $95 \%$ prediction limits for each farm.

A farmer would adopt the treatment if the lower $95 \%$ prediction limit on the difference between the predicted response of the treatment and the predicted response of his control on the farm is greater than zero. Results in Table 4 indicate that none of the farms should adopt the new treatment.

\subsection{Example 2}

A single treatment was compared against possibly different controls where ten farms were involved in the trial. The first six farms used a CRD and the rest used a RCBD. There were equal numbers of replications per farm. A general form of the model for CRD used on the six farms can be expressed as

$$
Y_{i j k}=\mu+f_{i}+\tau_{k}+f t_{i k}+\epsilon_{i j k}, \quad \mathrm{i}=1,2, \ldots, 6, \mathrm{j}=1,2, \ldots, 5, \mathrm{k}=\mathrm{T}, \mathrm{C}_{i}
$$

Similarly, a general model for the rest of the farms using RCBD is

$$
Y_{i j k}=\mu+f_{i}+r(f)_{i j}+\tau_{k}+f t_{i k}+\epsilon_{i j k}, \mathrm{i}=7,8,9,10, \mathrm{j}=1,2, \ldots, 5, \mathrm{k}=\mathrm{T}, \mathrm{C}_{i}
$$

All the terms in the above two models are the same as those defined in model (1) except for $r\left(f_{i j}\right.$ which represents the effect of the $j^{\text {th }}$ replication in the $i^{\text {th }}$ farm. Regardless of the model used, (9) remains the same and therefore, the estimate of $\mu_{s}$ and its estimated variance can be obtained as described in Example 1. In the analysis, we created a new blocking variable where the new block equals 1 if the data is from CRD and equals the replication number within the farm if data is from RCBD. This allows SAS/STAT software to predict an appropriate estimate of farmby- treatment interaction variance component. The contribution to the block within farm variance component, $\sigma_{b(\hat{)})}^{2}$ is zero for CRD whereas for RCBD is not.

The 1992 Sorghum data given in Table 5 were obtained from Cooperative Extension Service Program, Department of Agronomy, Kansas State university. The farms replications, treatments, blocks and yields are denoted by F, R, Trt, Blk and Yld, respectively. Table 6 contains restricted maximum likelihood estimates (REML) of variance components. The estimates of variance components obtained using the method of moments were used as the starting values in the iteration process using PROC MIXED (SAS, 1992). The estimates of variance components showed in Table 6 are used in the computations that are necessary for the estimation of $\mu_{s}$. 
Table 7 provides summarized results obtained for the estimation of $\mu_{s}$ computed in four ways, combining and computing with equal and unequal variances and when $\hat{\sigma}_{\tilde{n}}^{2}$ was either omitted or not omitted in the weighting process. From Table 7 , for unequal variance and $\hat{o}_{\tilde{f}}^{2}$ not omitted $\hat{\mu}_{s}=23.62, S . \hat{E}_{.}\left(\hat{\mu}_{s}\right)=9.913$, the approximated degrees of freedom are

$v_{\text {aver }}=(7.860+10.993) / 2=9.426 \approx 9$, and $t_{.05,9.426}=1.824$. A lower $95 \%$ confidence limit on $\hat{\mu}_{s}$ is $23.62-(1.824)(9.913)=5.54$. This treatment performs better than the mean of the population of farmers' controls since the lower $95 \%$ confidence limit on $\mu_{s}$ is greater than zero.

Table 8 shows the computations for the BLUPS of $U_{i T}-U_{i C_{i}}, i=1,2, \ldots, f$ and the estimated standard errors, and the lower $95 \%$ prediction limits for each farm. The results in Table 8 suggest that just farms 9 and 10 should adopt the new treatment.

\section{Summary and Conclusions}

A mixed model approach applies to the analysis of data from on-farm trials. The farm-bytreatment interaction variance, $\sigma_{f t}^{2}$ is assumed to be greater than zero unlike in meta-analysis where it is not necessarily interpretable. In on-farm trials, the same response is measured in all the farms under consideration and therefore, the farm-by-treatment interaction variance is meaningful and should be computed. Meta-analysis methods have wrongfully ignored farm-bytreatment interaction variance.

The rationale for testing the treatment against the mean of the controls is to recommend to the farmers the former once it performs better than the latter. A treatment is said to perform better than the mean of the population of farmers' controls if the lower $(1-\alpha) 100 \%$ confidence limit on $\mu_{s}$ is greater than zero. A lower $(1-\alpha) 100 \%$ prediction limit on each predictable function provides a narrow inference back to the single farmer. The information from the BLUP and prediction limit provides for the farmer the predicted performance of the treatment and assess how well it compares to the predicted performance of the control on the farm. Mixed model methods should be used for prediction rather than simple differences between means.

Regardless of the experimental design used in a given farm, all the random variables cancel out as a result of within farm comparisons, except for the farm-by-treatment interaction component and the within farm variance $\sigma_{i}^{2}, i=1,2, \ldots, f$. This point was demonstrated in Example 2, where a CRD was used at some farms and a RCBD was used on other farms. This fact makes our statistical procedure invariant with respect to the experimental design used at each farm. From both Example 1 and 2, we conclude that the estimate of farm-by-treatment interaction variance component is needed to be used in the weighting process for all on-farm trials. Unavailability of a common control only or a common experimental design on each farm should not be a hindrance to conducting and analyzing on-farm trials. 


\section{Acknowledgements}

The Authors are grateful to Dr. D.L. Devlin of Agronomy Department, Kansas State University for permission to use the data. We also wish to thank Dr. W. W. Stroup for his comments.

\section{References}

Amir, p., and Knipscheer, H.C., 1989. Conducting on-farm animal research procedures and economic analysis. Morrilton, ark: Winrock International Institute for Agricultural Development; International Development; International Development Research Centre.

Giesbrecht, F.G., and Burns, J.C., 1985. Two-stage analysis based on a mixed model: large-sample asymptotic theory and small-sample simulation results. Biometrics 41 , 477-486.

Hedges, L.V., 1981. Distribution theory for Glass's estimator of effect size and related estimators. Journal of Educational Statistics Vol. 6, Number 2, 107-128.

Hedges, L. and Olkin, I., 1985. Statistical methods for meta-analysis. Academic Press, Inc., New York.

Kacker, R.N., and Harville, D.A., 1984. Approximations for standard errors of fixed and random effects in mixed linear models. Journal of the American Statistical Association, 79, 853-862.

Li, Y. Li, S., and Roth, H.D., 1994. The bias of the commonly-used estimate of variance in meta-analysis. Commun. Statist.-Theory Meth., 23(4), 1063-1085.

Satterthwaite, F.F., 1941. Synthesis of variance. Psychometrika 6, 309-316.

SAS Technical report, SAS/STAT software; changes and enhancement release 6.0. SAS Institute, Inc., 1992. SAS Institute Inc., Cary, NC.

Stucker, R.E., and Hicks, D.R., 1993. Experimental design and plot size considerations for onfarm research. 46Th Annual Corn \& Sorghum Research Conference. 58-75. 
Table 1:Yield of Sorghum in 1991 (Bushel per Acre) for Example 1

\begin{tabular}{|c|c|c|c|c|c|c|c|c|c|c|c|}
\hline $\mathrm{Fm}$ & Rep & $\mathrm{Tm}$ & it Yld & $\mathrm{Fm}$ & Rep & $\mathrm{Tmt}$ & Yld & $\mathrm{Fm}$ & & $\operatorname{Tm}$ & it Yld \\
\hline 1 & 1 & $\mathrm{~T}$ & 74.4 & 3 & 1 & $\mathrm{~T}$ & 110.5 & 5 & 1 & $\mathrm{~T}$ & 143.4 \\
\hline 1 & 1 & $\mathrm{Cl}$ & 93.6 & 3 & 1 & C3 & 106.3 & 5 & 1 & C5 & 129.8 \\
\hline 1 & 2 & $\mathrm{~T}$ & 42.7 & 3 & 2 & $\mathrm{~T}$ & 91.2 & 5 & 2 & $\mathrm{~T}$ & 171.5 \\
\hline 1 & 2 & $\mathrm{Cl}$ & 79.7 & 3 & 2 & $\mathrm{C} 3$ & 95.0 & 5 & 2 & C5 & 119.4 \\
\hline 1 & 3 & $\mathrm{~T}$ & 61.1 & 3 & 3 & $\mathrm{~T}$ & 141.7 & 5 & 3 & $\mathrm{~T}$ & 135.7 \\
\hline 1 & 3 & $\mathrm{Cl}$ & 96.4 & 3 & 3 & $\mathrm{C} 3$ & 137.4 & 5 & 3 & C5 & 107.6 \\
\hline 1 & 4 & $\mathrm{~T}$ & 84.8 & 3 & 4. & $\mathrm{~T}$ & 88.5 & 5 & 4 & $\mathrm{~T}$ & 149.0 \\
\hline 1 & 4 & $\mathrm{Cl}$ & 104.9 & 3 & 4 & C3 & 101.5 & 5 & 4 & C5 & 145.1 \\
\hline 1 & 5 & $\mathrm{~T}$ & 68.7 & 3 & 5 & $\mathrm{~T}$ & 117.8 & 5 & 5 & $\mathrm{~T}$ & 140.8 \\
\hline 1 & 5 & $\mathrm{Cl}$ & 95.1 & 3 & 5 & $\mathrm{C} 3$ & 114.9 & 5 & 5 & $\mathrm{C} 5$ & 130.8 \\
\hline 2 & 1 & $\mathrm{~T}$ & 101.8 & 4 & 1 & $\mathrm{~T}$ & 165.6 & 6 & 1 & $\mathrm{~T}$ & 199.2 \\
\hline 2 & 1 & $\mathrm{C} 2$ & 114.2 & 4 & 1 & $\mathrm{C} 4$ & 138.1 & 6 & 1 & C6 & 154.3 \\
\hline 2 & 2 & $\mathrm{~T}$ & 92.7 & 4. & 2 & $\mathrm{~T}$ & 146.4 & 6 & 2 & $\mathrm{~T}$ & 192.5 \\
\hline 2 & 2 & $\mathrm{C} 2$ & 106.7 & 4. & 2 & $\mathrm{C} 4$ & 128.8 & 6 & 2 & C6 & 155.3 \\
\hline 2 & 3 & $\mathrm{~T}$ & 61.7 & 4 & 3 & $\mathrm{~T}$ & 153.0 & 6 & 3 & $\mathrm{~T}$ & 160.0 \\
\hline 2 & 3 & $\mathrm{C} 2$ & 88.7 & 4 & 3 & $\mathrm{C} 4$ & 121.1 & 6 & 3 & C6 & 136.4 \\
\hline 2 & 4 & $\mathrm{~T}$ & 109.1 & 4 & 4 & $\mathrm{~T}$ & 109.3 & 6 & 4 & $\mathrm{~T}$ & 175.9 \\
\hline 2 & 4 & $\mathrm{C} 2$ & 127.1 & 4 & 4 & $\mathrm{C} 4$ & 89.2 & 6 & 4 & C6 & 132.5 \\
\hline 2 & 5 & $\mathrm{~T}$ & 98.2 & 4 & 5 & $\mathrm{~T}$ & 131.8 & 6 & 5 & $\mathrm{~T}$ & 168.2 \\
\hline 2 & 5 & $\mathrm{C}_{2}$ & 113.5 & 4 & 5 & $\mathrm{C} 4$ & 128.6 & 6 & 5 & C6 & 140.6 \\
\hline
\end{tabular}

Table 2: The Estimates of Variance Component for the Random Effects for Example 1

\begin{tabular}{|c|l|l|}
\hline Random Effect & $\begin{array}{l}\text { Variance } \\
\text { Component }\end{array}$ & Estimate \\
\hline Farm & $\sigma_{f}^{2}$ & 683.452 \\
Farm*Treatment & $\sigma_{\tilde{t}}^{2}$ & 248.693 \\
Farm 1 & $\sigma_{1}^{2}$ & 166.003 \\
Farm 2 & $\sigma_{2}^{2}$ & 266.236 \\
Farm 3 & $\sigma_{3}^{2}$ & 370.270 \\
Farm 4 & $\sigma_{4}^{2}$ & 411.217 \\
Farm 5 & $\sigma_{5}^{2}$ & 195.057 \\
Farm 6 & $\sigma_{6}^{2}$ & 189.075 \\
Pooled Residual & $\sigma^{2}$ & 266.310 \\
\hline
\end{tabular}


Table 3: Estimates of the Means Difference and the Corresponding Standard Errors for Example 1 Sorghum Data.

\begin{tabular}{|l|ll|ll|}
\hline & \multicolumn{3}{|c|}{ Equal Variance } \\
& $\begin{array}{l}\text { Estimate } \\
\hat{\mu}_{s}\end{array}$ & $\begin{array}{l}\text { Standard Error } \\
\text { S.E. }\left(\hat{\mu}_{s}\right)\end{array}$ & $\begin{array}{l}\text { Estimate } \\
\hat{\mu}_{s}\end{array}$ & $\begin{array}{l}\text { Unequal Variance } \\
\text { S.E. }\left(\hat{\mu}_{s}\right)\end{array}$ \\
\hline$\hat{\sigma}_{\tilde{n}}^{2}$ omitted & 5.15 & 4.213 & 4.41 & 3.974 \\
\hline$\hat{\sigma}_{\mu t}^{2}$ not omitted & 5.15 & 10.032 & 5.02 & 10.013 \\
\hline
\end{tabular}

Table 4 :The BLUPS and the Lower $95 \%$ Prediction Limits for Each Farm for Example1

\begin{tabular}{|c|c|c|c|c|c|c|}
\hline $\begin{array}{c}\text { Farm } \\
\mathrm{i}\end{array}$ & $\hat{\eta}_{i}$ & $v_{i}$ & $\mathcal{S}_{i}$ & $\hat{t}_{0.05, v_{i}}$ & $\hat{S} \hat{E} .\left(S_{i}\right)$ & $\begin{array}{c}\text { Lower 95\% } \\
\text { Pred. Limit }\end{array}$ \\
\hline 1.0 & 563.788 & 4.320 & -27.60 & 2.083 & 23.744 & -77.06 \\
2.0 & 603.881 & 4.918 & -17.34 & 2.022 & 24.574 & -67.03 \\
3.0 & 645.495 & 5.553 & -1.08 & 1.971 & 25.407 & -51.16 \\
4.0 & 661.873 & 5.805 & 20.06 & 1.955 & 25.727 & -30.24 \\
5.0 & 575.409 & 4.492 & 21.54 & 2.066 & 23.988 & -28.02 \\
6.0 & 573.017 & 4.456 & 35.34 & 2.071 & 23.938 & -14.23 \\
\hline
\end{tabular}


Table 5: The 1991 Sorghum Data for Example 2 (Column 1-CRD, Column 2-RCBD)

\begin{tabular}{|c|c|c|c|c|c|c|c|c|c|c|c|c|c|c|}
\hline$F$ & $\mathrm{R}$ & $\mathrm{Tm}$ & & $1 \mathrm{~K}$ Yld & $\mathrm{F}$ & $\mathrm{R}$ & $\operatorname{Tm}$ & t & BIK YId & $\mathrm{F}$ & $\mathrm{R}$ & \multicolumn{3}{|c|}{ Tmt Blk Yld } \\
\hline 1 & 1 & $\mathrm{~T}$ & 1 & 63.5 & 5 & 1 & $\mathrm{~T}$ & 1 & 123.9 & 7 & 1 & $\mathrm{~T}$ & & 152.9 \\
\hline 1 & 1 & $\mathrm{C} 1$ & 1 & 94.3 & 5 & 1 & $\mathrm{C} 5$ & 1 & 99.7 & 7 & 1 & $\mathrm{C} 7$ & 1 & 151.1 \\
\hline 1 & 2 & $\mathrm{~T}$ & 1 & 75.1 & 5 & 2 & $\mathrm{~T}$ & 1 & 111.4 & 7 & 2 & $\mathrm{~T}$ & 2 & 202.9 \\
\hline 1 & 2 & $\mathrm{Cl}$ & 1 & 105.2 & 5 & 2 & $\mathrm{C} 5$ & 1 & 94.9 & 7 & 2 & $\mathrm{C} 7$ & 2 & 131.9 \\
\hline 1 & 3 & $\mathrm{~T}$ & 1 & 76.7 & 5 & 3 & $\mathrm{~T}$ & 1 & 136.6 & 7 & 3 & $\mathrm{~T}$ & 3 & 184.0 \\
\hline 1 & 3 & $\mathrm{Cl}$ & 1 & 107.6 & 5 & 3 & $\mathrm{C} 5$ & 1 & 132.8 & 7 & 3 & $\mathrm{C} 7$ & 3 & 143.7 \\
\hline 1 & 4 & $\mathrm{~T}$ & 1 & 86.8 & 5 & 4 & $\mathrm{~T}$ & 1 & 135.5 & 7 & 4 & $\mathrm{~T}$ & 4 & 190.8 \\
\hline 1 & 4 & $\mathrm{Cl}$ & 1 & 99.0 & 5 & 4 & $\mathrm{C5}$ & 1 & 103.5 & 7 & 4 & $\mathrm{C} 7$ & 4 & 155.4 \\
\hline 1 & 5 & $\mathrm{~T}$ & 1 & 85.2 & 5 & 5 & $\mathrm{~T}$ & 1 & 150.7 & 7 & 5 & $\mathrm{~T}$ & 5 & 212.5 \\
\hline 1 & 5 & $\mathrm{Cl}$ & 1 & 93.4 & 5 & 5 & $\mathrm{C} 5$ & 1 & 126.3 & 7 & 5 & $\mathrm{C} 7$ & 5 & 171.3 \\
\hline 2 & 1 & $\mathrm{~T}$ & 1 & 102.2 & 6 & 1 & $\mathrm{~T}$ & 1 & 175.0 & 8 & 1 & $\mathrm{~T}$ & 1 & 232.5 \\
\hline 2 & 1 & $\mathrm{C}_{2}$ & 1 & 115.3 & 6 & 1 & C6 & 1 & 138.5 & 8 & 1 & C8 & 1 & 183.7 \\
\hline 2 & 2 & $\mathrm{~T}$ & 1 & 94.2 & 6 & 2 & $\mathrm{~T}$ & 1 & 129.2 & 8 & 2 & $\mathrm{~T}$ & 2 & 215.5 \\
\hline 2 & 2 & $\mathrm{C}_{2}$ & 1 & 96.2 & 6 & 2 & C6 & 1 & 110.9 & 8 & 2 & C8 & 2 & 181.3 \\
\hline 2 & 3 & $\mathrm{~T}$ & 1 & 95.9 & 6 & 3 & $\mathrm{~T}$ & 1 & 128.3 & 8 & 3 & $\mathrm{~T}$ & 3 & 228.8 \\
\hline 2 & 3 & $\mathrm{C} 2$ & 1 & 113.4 & 6 & 3 & $\mathrm{C} 6$ & 1 & 92.9 & 8 & 3 & $\mathrm{C} 8$ & 3 & 178.8 \\
\hline 2 & 4 & $\mathrm{~T}$ & 1 & 98.8 & 6 & 4 & $\mathrm{~T}$ & 1 & 114.0 & 8 & 4 & $\mathrm{~T}$ & 4 & 214.2 \\
\hline 2 & 4 & $\mathrm{C} 2$ & 1 & 110.1 & 6 & 4 & C6 & 1 & 83.1 & 8 & 4 & $\mathrm{C} 8$ & 4 & 144.9 \\
\hline 2 & 5 & $\mathrm{~T}$ & 1 & 94.8 & 6 & 5 & $\mathrm{~T}$ & 1 & 131.0 & 8 & 5 & $\mathrm{~T}$ & 5 & 199.0 \\
\hline 2 & 5 & $\mathrm{C}_{2}$ & 1 & 101.2 & 6 & 5 & C6 & 1 & 80.0 & 8 & 5 & C8 & 6 & 149.3 \\
\hline 3 & 1 & $\mathrm{~T}$ & 1 & 112.7 & & & & & & 9 & 1 & $\mathrm{~T}$ & 1 & 197.2 \\
\hline 3 & 1 & $\mathrm{C} 3$ & 1 & 110.6 & & & & & & 9 & 1 & $\mathrm{Cg}$ & 1 & 152.5 \\
\hline 3 & 2 & $\mathrm{~T}$ & 1 & 107.0 & & & & & & 9 & 2 & $\mathrm{~T}$ & 2 & 207.5 \\
\hline 3 & 2 & C3 & 1 & 115.7 & & & & & & 9 & 2 & $\mathrm{Cg}$ & 2 & 149.2 \\
\hline 3 & 3 & $\mathrm{~T}$ & 1 & 120.4 & & & & & & 9 & 3 & $\mathrm{~T}$ & 3 & 209.2 \\
\hline 3 & 3 & $\mathrm{C} 3$ & 1 & 127.9 & & & & & & 9 & 3 & $\mathrm{CP}$ & 3 & 154.1 \\
\hline 3 & 4 & $\mathrm{~T}$ & 1 & 110.9 & & & & & & 9 & 4 & $\mathrm{~T}$ & 4 & 237.5 \\
\hline 3 & 4 & $\mathrm{C} 3$ & 1 & 99.8 & & & & & & 9 & 4 & $\mathrm{CP}$ & 4 & 180.2 \\
\hline 3 & 5 & $\mathrm{~T}$ & 1 & 102.4 & & & & & & 9 & 5 & $\mathrm{~T}$ & 5 & 244.0 \\
\hline 3 & 5 & $\mathrm{C} 3$ & 1 & 105.8 & & & & & & 9 & 5 & $\mathrm{Cg}$ & 5 & 176.1 \\
\hline 4 & 1 & $\mathrm{~T}$ & 1 & 142.4 & & & & & & 10 & 1 & $\mathrm{~T}$ & 1 & 253.7 \\
\hline 4 & 1 & $\mathrm{C} 4$ & 1 & 134.7 & & & & & & 10 & 1 & $\mathrm{ClO}$ & 1 & 193.6 \\
\hline 4 & 2 & $\mathrm{~T}$ & 1 & 128.6 & & & & & & 10 & 2 & $\mathrm{~T}$ & 2 & 261.8 \\
\hline 4 & 2 & $\mathrm{C} 4$ & 1 & 127.5 & & & & & & 10 & 2 & $\mathrm{ClO}$ & 2 & 202.0 \\
\hline 4 & 3 & $\mathrm{~T}$ & 1 & 137.2 & & & & & & 10 & 3 & $\mathrm{~T}$ & 3 & 262.3 \\
\hline 4 & 3 & $\mathrm{C} 4$ & 1 & 138.3 & & & & & & 10 & 3 & $\mathrm{ClO}$ & 3 & 161.3 \\
\hline 4 & 4 & $\mathrm{~T}$ & 1 & 111.5 & & & & & & 10 & 4 & $\mathrm{~T}$ & 4 & 205.9 \\
\hline 4 & 4 & $\mathrm{C} 4$ & 1 & 104.3 & & & & & & 10 & 4 & $\mathrm{C} 10$ & 4 & 143.9 \\
\hline 4 & 5 & $\mathrm{~T}$ & 1 & 101.8 & & & & & & 10 & 5 & $\mathrm{~T}$ & 5 & 225.5 \\
\hline 4 & 5 & $\mathrm{C} 4$ & 1 & 87.0 & & & & & & 10 & 5 & $\mathrm{C} 10$ & 5 & 149.0 \\
\hline
\end{tabular}


Table 8: The Estimates of Variance Components for the Random Effects for Example 2

\begin{tabular}{|l|l|r|}
\hline $\begin{array}{l}\text { Random } \\
\text { Effect }\end{array}$ & $\begin{array}{l}\text { Variance } \\
\text { Component }\end{array}$ & Estimate \\
\hline Farm & $\sigma_{f}^{2}$ & 1432.373 \\
Block(Farm) & $\sigma_{b(f)}^{2}$ & 230.680 \\
Farm*Treatment & $\sigma_{\pi}^{2}$ & 435.884 \\
Farm 1 & $\sigma_{1}^{2}$ & 63.746 \\
Farm 2 & $\sigma_{2}^{2}$ & 39.182 \\
Farm 3 & $\sigma_{3}^{2}$ & 79.555 \\
Farm 4 & $\sigma_{4}^{2}$ & 388.984 \\
Farm 5 & $\sigma_{5}^{2}$ & 252.982 \\
Farm 6 & $\sigma_{6}^{2}$ & 490.091 \\
Farm 7 & $\sigma_{7}^{2}$ & 302.714 \\
Farm 8 & $\sigma_{8}^{2}$ & 77.858 \\
Farm 9 & $\sigma_{9}^{2}$ & 34.364 \\
Farm 10 & $\sigma_{10}^{2}$ & 156.453 \\
Pooled Residual & $\sigma^{2}$ & 200.029 \\
\hline
\end{tabular}

Table 10: Estimates of the Means Difference and the Corresponding Standard Errors for Example 2 Sorghum Data.

\begin{tabular}{|l|ll|ll|}
\hline & \multicolumn{2}{|c|}{ Equal Variance } \\
Estimate & $\begin{array}{l}\text { Standard Error } \\
\text { S.E. }\left(\hat{\mu}_{s}\right)\end{array}$ & \multicolumn{2}{c|}{$\begin{array}{l}\text { Unequal Variance } \\
\hat{\mu}_{s}\end{array}$} & $\begin{array}{l}\text { Standard Error } \\
\text { S.E. }\left(\hat{\mu}_{s}\right)\end{array}$ \\
\hline$\hat{\sigma}_{\tilde{f t}}^{2}$ omitted & 23.76 & 2.829 & 21.14 & 1.873 \\
\hline$\hat{\sigma}_{f t}^{2}$ not omitted & 23.76 & 9.756 & 23.62 & 9.713 \\
\hline
\end{tabular}


Table 8: The BLUPS and the Lower 95\% Prediction Limits for Each Farm for Example2

\begin{tabular}{|c|c|c|c|c|c|c|}
\hline $\begin{array}{c}\text { Farm } \\
\mathrm{i}\end{array}$ & $\hat{\eta}_{i}$ & $v_{i}$ & $S_{i}$ & $t_{0.05, v_{i}}$ & $S . \hat{E}\left(S_{i}\right)$ & $\begin{array}{c}\text { Lower 95\% } \\
\text { Pred. Limit }\end{array}$ \\
\hline 1.0 & 897.27 & 8.066 & -22.44 & 1.857 & 29.954 & -78.06 \\
2.0 & 887.44 & 7.894 & -10.06 & 1.863 & 29.790 & -65.56 \\
3.0 & 903.59 & 8.176 & -1.28 & 1.854 & 30.060 & -57.01 \\
4.0 & 1027.36 & 10.319 & 5.94 & 1.807 & 32.054 & -51.98 \\
5.0 & 972.96 & 9.387 & 20.18 & 1.824 & 31.192 & -36.71 \\
6.0 & 1067.80 & 10.993 & 28.42 & 1.796 & 32.677 & -30.27 \\
7.0 & 992.85 & 9.731 & 37.94 & 1.817 & 31.509 & -19.31 \\
8.0 & 902.91 & 8.164 & 50.40 & 1.855 & 30.048 & -5.34 \\
9.0 & 885.51 & 7.860 & 56.66 & 1.864 & 29.758 & 1.19 \\
10.0 & 934.35 & 8.714 & 71.88 & 1.840 & 30.567 & 15.64 \\
\hline
\end{tabular}

